CTP TAMU-10/00

$\mathrm{UPR} / 881-\mathrm{T}$

March 2000

hep-th/0003286

\title{
Consistent Kaluza-Klein Sphere Reductions
}

\author{
M. Cvetič ${ }^{\dagger 1}$, H. Lü ${ }^{\dagger 1}$ and C.N. Pope ${ }^{\ddagger 2}$ \\ ${ }^{\dagger}$ Dept. of Phys. and Astro., University of Pennsylvania, Philadelphia, PA 19104 \\ ${ }^{\ddagger}$ Center for Theoretical Physics, Texas A $ॄ$ M University, College Station, TX 77843
}

\begin{abstract}
$\underline{\text { ABSTRACT }}$
We study the circumstances under which a Kaluza-Klein reduction on an $n$-sphere, with a massless truncation that includes all the Yang-Mills fields of $S O(n+1)$, can be consistent at the full non-linear level. We take as the starting point a theory comprising a $p$-form field strength and (possibly) a dilaton, coupled to gravity in the higher dimension $D$. We show that aside from the previously-studied cases with $(D, p)=(11,4)$ and $(10,5)$ (associated with the $S^{4}$ and $S^{7}$ reductions of $D=11$ supergravity, and the $S^{5}$ reduction of type IIB supergravity), the only other possibilities that allow consistent reductions are for $p=2$, reduced on $S^{2}$, and for $p=3$, reduced on $S^{3}$ or $S^{D-3}$. We construct the fully non-linear Kaluza-Klein Ansätze in all these cases. In particular, we obtain $D=3, N=8, S O(8)$ and $D=7, N=2, S O(4)$ gauged supergravities from $S^{7}$ and $S^{3}$ reductions of $N=1$ supergravity in $D=10$.
\end{abstract}

\footnotetext{
${ }^{1}$ Research supported in part by DOE grant DOE-FG02-95ER40893

${ }^{2}$ Research supported in part by DOE grant DOE-FG03-95ER40917
} 


\section{Introduction}

Much progress has been achieved recently in understanding the full non-linear structure of certain Kaluza-Klein sphere reductions. To be specific, we have in mind the remarkable cases where it is consistent to include lower-dimensional fields in the reduction Ansatz that parameterise inhomogeneous deformations of the internal sphere metric. Generically, one would expect that performing a truncation of the complete Kaluza-Klein towers of massless and massive modes to the purely massless sector would give rise to inconsistencies beyond the linearised level, since currents built from the massless fields would act as sources for the massive fields that have been set to zero. Indeed this is exactly what usually happens; one cannot make a consistent Kaluza-Klein $n$-sphere reduction of a generic theory in which all the massless fields, including, in particular, the full set of $S O(n+1)$ gauge fields are retained. However, in certain very special cases a consistent reduction of this kind is possible.

An important early example of this type was much studied in the 1980's, namely the seven-sphere compactification of eleven-dimensional supergravity. It was first shown at the level of linearised fluctuations [1] around the $\mathrm{AdS}_{4} \times S^{7}$ Freund-Rubin [2] vacuum solution that the massless modes described four-dimensional $N=8$ gauged $S O(8)$ supergravity [3]. Subsequently, it was shown that despite all the apparent obstacles, the reduction to the massless $N=8$ multiplet can be carried through as an exact embedding at the full non-linear level [4], although the construction is an extremely complex one. It has long been believed that consistent reductions should also be possible in the case of the $S^{5}$ compactification of type IIB supergravity, and the $S^{4}$ compactification of eleven-dimensional supergravity, to yield the maximal gauged $S O(6)$ supergravity in $D=5$, and the maximal gauged $S O(5)$ supergravity in $D=7$, respectively. Indeed, the consistent $S^{4}$ reduction Ansatz from $D=11$ has recently been constructed [5, 6]. No analogous construction exists for the complete massless reduction on $S^{5}$.

It is sometimes helpful to study sphere reductions in which only a subset of the complete set of massless fields is retained, in such a way that one still has the non-triviality of the inhomogeneous sphere deformations, while at the same time making the problem of obtaining an explicit reduction Ansatz more tractable and manageable. This can be espe-

cially useful if one actually wants to use the Ansatz for the purpose of lifting solutions of the lower-dimensional theory back to the higher dimension, in which case full explicit reduction formulae are highly advantageous. In this spirit, consistent reductions in the three cases mentioned above were constructed for truncations in which only the maximal abelian subgroups $U(1)^{4}, U(1)^{3}$ and $U(1)^{2}$ of the full $S O(8), S O(6)$ and $S O(5)$ gauge groups were 
retained, together with associated scalar fields [7]. The $U(1)^{3}$ example provided the first concrete evidence for the consistency of the $S^{5}$ reduction of type IIB supergravity. The reduction Ansätze were sufficiently explicit that they could be used for the purpose of lifting certain AdS black-hole solutions back to the higher dimension [7], where they become rotating D3-branes [8, 9, 10, 7] and M-branes [11, 7].

Other consistent reductions involving subsets of the complete massless sector have subsequently been constructed, including an $S^{4}$ reduction to give $N=2$ gauged $S U(2)$ supergravity in $D=7$ [12]; an $S^{5}$ reduction to $N=4$ gauged $S U(2) \times U(1)$ supergravity in $D=5$ [13]; and an $S^{7}$ reduction to $N=4$ gauged $S O(4)$ supergravity in $D=4$ [14. In addition, the $N=2$ gauged $S U(2)$ supergravity in $D=6$ was obtained via a consistent reduction from massive type IIA supergravity on a locally $S^{4}$ internal space 15. This is actually the largest possible supersymmetry for a gauged theory in $D=6$, even though the maximum supersymmetry for ungauged supergravity is $N=4$.

Somtimes, It can also be useful to construct a Kaluza-Klein sphere reduction in which a non-supersymmetric truncation of the massless supermultiplet is made. One example of this type involved truncating the maximal supergravities in $D=4,5$ and 7 to a subsector comprising just gravity and a set of 7, 5 or 4 scalars respectively. These scalars correspond to the diagonal subset of fields in the unimodular symmetric tensors $T_{i j}$ describing the scalars in the $S L(8, \mathbb{R}) / S O(8), S L(6, \mathbb{R}) / S O(6)$ and $S L(5, \mathbb{R}) / S O(5)$ scalar submanifolds of the full supergravities. In [16, 17], the full non-linear reduction Ansätze for these embedding were constructed, and proved to be consistent.

Another example of a non-supersymmetric truncation was constructed in [18], where the full set of twenty scalars $T_{i j}$ of the coset $S L(6, \mathbb{R}) / S O(6)$ were retained in an $S^{5}$ reduction from $D=10$. Consistency now requires that one include also the full set of $S O(6)$ YangMills gauge fields. In fact only the metric and self-dual 5-form of the type IIB supergravity are involved in this reduction, and it can equivalently be viewed as a Kaluza-Klein reduction of a theory of pure gravity plus self-dual 5 -form in $D=10$, with all massless fields retained in $D=5$. (The truncation of type IIB supergravity to just gravity and the self-dual 5form is itself a consistent one in $D=10$.) The self-duality of the 5-form is crucial for the consistency of the reduction.

One should not conclude from the listing of examples above that consistent Kaluza-Klein sphere reductions are a commonplace. In fact, if we restrict attention to cases where one starts in the higher dimension with just gravity and a $p$-form field strength, then it turns out that the only cases that can give consistent reductions are related to the examples mentioned 
above. The reason for this can be understood as follows. For reductions of the type we are considering, where the lower-dimensional theory obtained by the $S^{n}$ reduction has an $S O(n+1)$ local gauge symmetry, it is essential that the ungauged theory that would result from performing a reduction on the $n$-torus rather than the $n$-sphere should have scalars described by a coset manifold $G / H$ such that $H$ at least contains $S O(n+1)$. The reason for this is that in the process of gauging the ungauged theory, a subgroup $S O(n+1)$ of the global symmetry $G$ must become local, and this subgroup must be contained within $H$. Now if a generic theory of gravity and antisymmetric tensors is reduced on $T^{n}$, it will give rise to a lower-dimensional theory with a $G L(n, \mathbb{R})$ global symmetry [19, 20, 21], for which the maximal compact subgroup is $S O(n)$. This is insufficient for allowing an $S O(n+1)$ gauging. Note that in particular this argument shows that it is not possible to perform a consistent $n$-sphere reduction of a pure gravity theory, in which the Yang-Mills fields of $S O(n+1)$ are retained.

In certain very special theories, the $G L(n, \mathbb{R})$ global symmetry arising from a $T^{n}$ reduction is enhanced to a larger symmetry, as a result of "conspiracies" between scalars coming from the Kaluza-Klein reduction of the metric and of the other higher-dimensional fields. However, as discussed in 21, 22], such cases are very few and far between. In particular, if we consider a $D$-dimensional theory consisting of gravity and a single $p$-form field strength, an enhancement of the global symmetry can occur only if $(D, p)$ is equal to $(11,4),(11,7)$ or $(10,5)$. Since a 7 -form in $D=11$ is dual to a 4 -form this means that the only cases with symmetry enhancements are associated with $D=11$ supergravity and type IIB supergravity. The corresponding enhanced symmetries in each case are to $S L(8, \mathbb{R}) / S O(8)$ in $D=4, S L(5, \mathbb{R}) / S O(5)$ in $D=7$, and $S L(6, \mathbb{R}) / S O(6)$ in $D=5$.2 These enhancements then allow the $S O(8), S O(6)$ and $S O(5)$ gaugings, respectively $]^{3}$

Note that whereas there is a consistent $S^{5}$ reduction of gravity with a self-dual 5 -form in

\footnotetext{
${ }^{1}$ To be precise, we should emphasise that what we are discussing here is cases where all of the $S O(n+1)$ Yang-Mills gauge fields associated with the isometry group of the $n$-sphere are retained in the truncation, together with other associated massless scalars.

${ }^{2}$ Actually further conditions must be fulfilled in order for the symmetry enhancements to take place. In the case of $D=11$ reduced to $D=4$ on $T^{7}$, the enhancement of $G L(7, \mathbb{R})$ to $S L(8, \mathbb{R})$ requires that the 4-form have an FFA term in $D=11$ with precisely the coefficient dictated by supersymmetry; this in fact means that the enhanced symmetry is even larger, namely $E_{7}$. For the $D=10$ theory reduced on $T^{5}$, the enhancement from $G L(5, \mathbb{R})$ to $S L(6, \mathbb{R})$ requires that the 5 -form be self-dual (or anti-self-dual).

${ }^{3}$ The importance of enhancements of the global symmetry in toroidal reductions was also observed in [23], although it was assumed there that the phenomenon was much more widespread than is actually the case.
} 
which only the metric, the $S O(6)$ Yang-Mills fields $A_{(1)}^{i j}$ and the 20 scalars $T_{i j}$ are retained, the situation is a little different in the $S^{4}$ and $S^{7}$ reductions from $D=11$. In addition to keeping the corresponding Yang-Mills fields $A_{(1)}^{i j}$ and scalars $T_{i j}$, the consistency of the $S^{4}$ reduction requires also keeping the five 3 -forms $A_{(3)}^{i}$ of the seven-dimensional theory, whilst the $S^{7}$ reduction instead requires also keeping the 35 pseudoscalars $\phi_{[i j k \ell]_{+}}$(self-dual in the $S O(8)$ indices). These additional fields are needed in the reductions because the Yang-Mills fields act as sources for them [18]. In fact we can summarise the situation in all three of these examples of the $S^{4}, S^{7}$ and $S^{5}$ reductions as follows. In all cases, the consistent $n$ sphere reduction that includes all the Yang-Mills fields of $S O(n+1)$ requires one to include all the massless fields in the lower-dimensional theory. Thus in the $S^{5}$ case, if we reduce the theory of gravity and the self-dual 5-form then the metric, the $S O(6)$ Yang-Mills fields and the scalars $T_{i j}$ indeed constitute the complete set of massless fields in five dimensions. In the $S^{4}$ reduction from $D=11$ the five 3 -forms $A_{(3)}^{i}$ are massless too, and indeed they must be included also in the consistent reduction. Likewise, in the $S^{7}$ reduction from $D=11$ the 35 pseudoscalars $\phi_{[i j k \ell]_{+}}$are also massless, and indeed they must be included in the consistent reduction.

Further possibilities for consistent $n$-sphere reductions in which all the $S O(n+1)$ YangMills fields are retained can arise if we consider a slightly enlarged higher-dimensional theory, now with a dilatonic scalar as well as gravity and the $p$-form field strength. Again, the key point is that an enhancement of the $G L(n, \mathbb{R})$ global symmetry that would occur for the reduction of a generic theory on $T^{n}$ is needed, in order that the scalar coset manifold in the lower dimension should have a denominator group that is large enough to contain the desired $S O(n+1)$ local symmetry group of the theory reduced on $S^{n}$. It turns out that by including the dilatonic scalar in the higher dimension, the necessary symmetry enhancements can be achieved when the $p$-form field strength is either a 2-form, or a 3-form. This opens up the possibility of finding consistent Kaluza-Klein reductions on $S^{2}$, for the 2-form case, and on $S^{3}$ or on $S^{D-3}$, for the 3 -form case. These, together with the previous $D=11$ and $D=10$ examples, would then constitute the complete list of possibilities for consistent Kaluza-Klein $n$-sphere reductions within the class of theories we are considering, in which all the Yang-Mills fields of $S O(n+1)$ are retained.

In this paper, we construct the complete and explicit non-linear Kaluza-Klein Ansätze for these three new possibilities. We begin in section 2 with a detailed discussion of the global symmetry enhancements that can occur in the toroidal reductions of theories with gravity, a $p$-form field strength and a dilaton, in order to establish what are the possibilities 
for consistent sphere reduction. In section 3 we construct the Ansatz for the consistent reduction of gravity plus a 3 -form and a dilaton on $S^{3}$, keeping all the gauge fields of $S O(4)$ and the ten scalars of the symmetric tensor $T_{i j}$, together with the 2 -form potential $A_{(2)}$. In section 4 , we examine two truncations of the $S^{3}$ reduction, in which only certain subsets of the massless fields are retained, in order to make contact with previous results in the literature. In section 5 we consider the "dual" of the $S^{3}$ reduction namely, the reduction instead on $S^{D-3}$. Again, we find a consistent reduction Ansatz, in which all the gauge fields of $S O(D-2)$ are retained, together with the $\frac{1}{2}(D-1)(D-2)$ scalars in $T_{i j}$. A case of particular interest is the $S^{7}$ reduction from $D=10$, since then the resulting threedimensional theory is the bosonic sector of a gauged $S O(8)$ supergravity, of a type not previously constructed in the literature. In section 6 we construct the consistent KaluzaKlein Ansatz for the reduction of a theory of gravity, a dilaton and a 2-form field strength on $S^{2}$. In this case the Ansatz includes all three gauge fields of $S O(3)$, together with the six scalars in $T_{i j}$.

Note that in two of the new cases that we consider here, namely the $S^{2}$ reduction of the theory with a 2 -form field strength, and the $S^{D-3}$ reduction of the theory with a 3 -form field strength, the totality of massless fields in the Kaluza-Klein reduced lower-dimensional theories comprise the metric, the Yang-Mills gauge fields $A_{(1)}^{i j}$, and the scalars $T_{i j}$. Thus these new examples of consistent sphere reductions are akin to the $S^{5}$ reduction of gravity plus a self-dual 5-form, in that no additional massless fields are present that must also be included in the reduction Ansatz. By constrast, in the new $S^{3}$ reduction that we construct here, we must additionally include the 2 -form potential $A_{(2)}$ in the Ansatz. This is similar to the situation for the $S^{4}$ and $S^{7}$ reductions, where, as we discussed previously, additional massless fields are present, and must be included, for consistency.

\section{Possibilities for $S O(n+1)$ Kaluza-Klein reductions on $S^{n}$}

As we mentioned in the Introduction, a consistent Kaluza-Klein reduction on $S^{n}$ that retains all the gauge fields of $S O(n+1)$ will be possible only if there is a suitable enhancement of the generic $G L(n, \mathbb{R})$ global symmetry that arises instead in a $T^{n}$ reduction, so that the denominator group of the generic scalar manifold $G L(n, \mathbb{R}) / O(n)$ becomes large enough to contain $S O(n+1)$. We also mentioned that there are only rather limited circumstances under which these symmetry enhancements can occur.

The reason why the possibilities for symmetry enhancements are so restrictive is dis- 
cussed extensively in [21, 22]. The scalars divide into $n$ "dilatons," $\vec{\phi}$ coming from the diagonal metric components of the internal $n$-torus, with the rest being "axions" $\chi_{i}$ coming from the off-diagonal metric components and the reduction of the antisymmetric tensor. Each dilaton has a kinetic term of the form $-\frac{1}{2} e^{\vec{c}_{i}} \cdot \vec{\phi}\left(\partial \chi_{i}\right)^{2}$, where $\vec{c}_{i}$ is the associated constant "dilaton vector" characterising the coupling of the dilatons to that particular axion.

In the $n$-torus reduction of a theory of $D$-dimensional gravity plus $p$-form field strength with general values of $D$ and $p$, the global symmetry will be $G L(n, \mathbb{R})$. In fact the dilaton vectors $\vec{b}_{i}$ associated with the axions coming from the metric form the complete set of positive roots of the $S L(n, \mathbb{R})$ algebra [21, 22]. The dilaton vectors $\vec{a}_{i}$ associated with the axions coming from the $p$-form field strength then form the weights of some representation under $S L(n, \mathbb{R})$. If an enhancement of the global symmetry is to occur, it must be that some or all of the dilaton vectors $\vec{a}_{i}$ "conspire" to become the additional positive roots of the enhanced symmetry algebra. However, this cannot occur in general, because the lengths of vectors $\vec{a}_{i}$ coming from the $p$-form will be incommensurate with the lengths of the vectors $\vec{b}_{i}$ coming from the metric.f

A convenient way to characterise the lengths of the various dilaton vectors was introduced in [24]. Rather than using the quantity $|\vec{c}|^{2}$ itself, it is convenient to introduce a constant $\delta$, related to $|\vec{c}|^{2}$ by

$$
|\vec{c}|^{2}=\delta-\frac{2(m-1)(D-m-1)}{D-2},
$$

where $D$ is the spacetime dimension, and $m$ is the degree of the field-strength whose dilaton coupling is $e^{\vec{c} \cdot \vec{\phi}}$. (Note that the all the field strengths in the $D=11$ and $D=10$ supergravities have $\delta=4$ couplings [25].) The key point about this parameterisation is that $\delta$ is preserved under toroidal Kaluza-Klein reduction. This makes it rather easy to see when the possibility of an enhancement of the global symmetry can occur. First, we note 24] that the dilaton vectors $\vec{b}_{i}$ associated with the axions coming from the Kaluza-Klein reduction of the metric always have $\delta=4$. It follows therefore that if the dilaton vectors $\vec{a}_{i}$ associated with axions coming from the $p$-form field strength in the higher dimension are to have the same lengths as the $\vec{b}_{i}$, then the coupling of the field strength must also have $\delta=4$. If we

\footnotetext{
${ }^{4}$ We first, of course, establish a canonical normalisation for the dilaton kinetic terms, so that comparisons of the lengths are meaningful.

${ }^{5}$ Since all the $\vec{a}_{i}$ themselves have equal length, and all the $\vec{b}_{i}$ themselves have equal length, it follows that to get a simply-laced enhanced symmetry group we would need that the length of the $\vec{a}_{i}$ and the length of the $\vec{b}_{i}$ should be equal. This turns out to be the only situation where relevant symmetry enhancements occur, within the framework of the higher-dimensional Lagrangians (3). Thus although the case $D=6$ with
} 
are starting in $D$ dimensions with a theory with just gravity and the $p$-form field strength, but no dilaton, this means that in $D$ dimensions we have $\vec{c}=0$, and $m=p$, and so to have $\delta=4$ we must have

$$
2 D-4=(p-1)(D-p-1) .
$$

It is easily verified that the only integer solutions are $(D, p)=(11,4),(11,7)$ and $(10,5)$.

The possibilities for achieving the necessary enhancement of the global $G L(n, \mathbb{R})$ symmetry can be broadened considerably if a dilatonic scalar is included in the original theory in $D$ dimensions, since now there is the possibility of adjusting its coupling to the $p$-form field strength so that the corresponding value of $\delta$ is equal to 4 . Thus we may consider the $D$-dimensional Lagrangian

$$
\mathcal{L}_{D}=\hat{R} \hat{*} \mathbb{1}-\frac{1}{2} \hat{*} d \hat{\phi} \wedge d \hat{\phi}-\frac{1}{2} e^{-a \hat{\phi}} \hat{*} \hat{F}_{(p)} \wedge \hat{F}_{(p)},
$$

with $a$ chosen so that

$$
a^{2}=4-\frac{2(p-1)(D-p-1)}{D-2} .
$$

Note that we put hats on all the fields in (3), to indicate that they are higher-dimensional quantities.

The first point to notice is that the requirement that the constant $a$ should be real is a rather restrictive one, since it implies

$$
p^{2}-3 D p+3 D-5 \geq 0 .
$$

Taken together with the fact that obviously $p$ cannot exceed $D$, this implies that the only additional possibilities opened up by the inclusion of the dilaton are for $p=2,3,(D-2)$ or $(D-3)$. The last two here are just the Hodge duals of $p=2$ and $p=3$, so we need not consider them as distinct cases. For $p=2$ and $p=3$, the relation (41) gives

$$
\begin{array}{ll}
p=2: & a^{2}=\frac{2(D-1)}{D-2}, \\
p=3: & a^{2}=\frac{8}{D-2} .
\end{array}
$$

a $\delta=2$ self-dual 3-form gives an enhancement to $O(3,4)$ after a $T^{3}$ reduction to $D=3$ [22], and $D=5$ with a $\delta=\frac{4}{3}$ 2-form gives an enhancement to $G_{2}$ after a $T^{2}$ reduction to $D=3$ [26, 22], neither of these non-simply-laced cases would seem to indicate the possibility of consistent $S^{3}$ or $S^{2}$ reductions.

${ }^{6}$ One might in principle consider also the possibility that $a$ could be imaginary. This would be equivalent to having a ghost-like kinetic term for the dilaton in the $D$-dimensional theory. This could not lead to any useful global symmetry enhancements from the point of view of sphere reductions that retain the $S O(n+1)$ gauge fields. There might be possible implications for consistent reductions on spaces with non-compact symmetry groups. 
Thus we see that if we start in $D$ dimensions with the Lagrangian (3) with a 2-form or 3 -form field strength, we can achieve a $\delta=4$ dilaton coupling in any dimension $D$, and thus we can expect to find an enhancement of the $G L(n, \mathbb{R})$ global symmetry after dimensional reduction on $T^{n}$. Indeed this is the case.

First, let us consider the case $p=3$, where we make a $T^{n}$ reduction of (3) with $a$ given by $(\overline{7})$. The global symmetry is indeed enhanced, and the scalar manifold in $(D-n)$ dimensions will be 27, 28, 22

$$
\begin{array}{ll}
D-n>3: & \mathbb{R} \times \frac{O(n, n)}{O(n) \times O(n)}, \\
D-n=3: & \frac{O(D-2, D-2)}{O(D-2) \times O(D-2)} .
\end{array}
$$

This $p=3$ case corresponds precisely to the T-duality symmetry of the toroidally-reduced bosonic string. Note that if $D-n=3$ the usual T-duality group $O(D-3, D-3)$ of the string theory reduced on $T^{n}$ is further enhanced to the non-perturbative U-duality group $O(D-2, D-2)$. Using the 3 -form field strength, we can then consider either an $S^{3}$ or an $S^{D-3}$ Kaluza-Klein reduction.

If we take $n=3$, we see that the the scalar coset manifold from a $T^{3}$ reduction will be

$$
\mathbb{R} \times \frac{O(3,3)}{O(3) \times O(3)} \sim \mathbb{R} \times \frac{S L(4, \mathbb{R})}{S O(4)} .
$$

There will also be six gauge potentials coming from the Kaluza-Klein reduction on $T^{3}$. This implies that the $S O(4)$ subgroup of the $S L(4, \mathbb{R})$ global symmetry group can be gauged, with the six vector potentials becoming the Yang-Mills fields of $S O(4)$. It is then natural to conjecture that this gauged theory may be directly obtainable as a Kaluza-Klein reduction on $S^{3}$. It is far from obvious that such a reduction would be consistent, since unlike the toroidal reduction there is no obvious group-theoretic argument that would guarantee the consistency at the non-linear level. In the next section, we shall explicitly show that the reduction on $S^{3}$, in which the full set of $S O(4)$ gauge fields are retained, is in fact consistent at the full non-linear level.

Now let us consider instead the $T^{D-3}$ reduction of (3), again with $p=3$. The reduced theory will now be in three dimensions, and the scalar coset manifold will be given by

\footnotetext{
${ }^{7}$ If we were gauging only the left-acting $S U(2)$ or only the right-acting $S U(2)$ of the $S O(4) \sim S U(2)_{L} \times$ $S U(2)_{R}$ isometry of the 3 -sphere (which is itself the group manifold $S U(2)$ ), then the consistency would be guaranteed, since the retained fields would then all be singlets under the other $S U(2)$, but this is no longer the case when the gauge fields of the full isometry group are retained. In fact we shall discuss the truncation to a single $S U(2)$ subgroup in section 4 .
} 
(9), provided that $a$ satisfies (7). Note that the further symmetry enhancement of this $D-n=3$ case occurs because the complete field content of the resulting three-dimensional theory (except for the metric) can be described by scalars, since in three dimensions one can dualise all the vector potentials to scalars. The coset (9) can also be described as [21]

$$
\frac{G L(D-2, \mathbb{R})}{O(D-2)} \ltimes V
$$

where $V$ is an irreducible representation under $G L(D-2, \mathbb{R})$ of dimension $\frac{1}{2}(D-2)(D-3)$; this is the same as the dimension of the adjoint representation of $O(D-2)$.

The scalars in the representation $V$ can be dualised to vector potentials. ${ }^{8}$ suggesting that the $O(D-2)$ denominator group in (11) can be gauged. Then we may conjecture that this gauged three-dimensional theory can alternatively be obtained as a reduction of the original $D$-dimensional theory on the sphere $S^{D-3}$. In section 5 , we shall demonstrate that there is indeed such a consistent reduction on $S^{D-3}$, in which all the gauge fields $A_{(1)}^{i j}$ of $O(D-2)$ are retained, together with scalars described by the symmetric tensor $T_{i j}$, where $i$ is a vector index of $O(D-2)$.

Finally, let us consider the Lagrangian (3) with $p=2$, where the dilaton coupling for the 2-form is given by (6). The Lagrangian (3) is then in fact precisely the $S^{1}$ dimensional reduction of pure gravity in $D+1$ dimensions. Consequently, the scalar manifold in $(D-n)$ dimensions after reducing (3) on $T^{n}$ will be enhanced to

$$
\frac{G L(n+1, \mathbb{R})}{S O(n+1)}
$$

With a 2-form field strength we have in principle two possibilities for sphere reductions, namely on $S^{2}$ or on $S^{D-2}$. The latter would be somewhat degenerate, since the lowerdimensional theory would be in $D=2$, so we shall just consider the $S^{2}$ possibility here. If we take $n=2$, the denominator group in (12) is exactly what is needed to allow an $S O(3)$ gauging. We may then conjecture that this gauged theory should alternatively be directly obtainable as a consistent Kaluza-Klein reduction on $S^{2}$, keeping all three of the $S O(3)$ Yang-Mills gauge fields, together with six scalars described by the symmetric tensor $T_{i j}$. We shall in fact construct this consistent reduction in section 6 .

We conclude this section with a Table that summarises all the cases where consistent sphere reductions of a $D$-dimensional theory comprising gravity, a $p$-form field strength and

\footnotetext{
${ }^{8}$ The description (11) would arise naturally if one dualised the $(D-3)$ vector potentials coming from the direct reduction of the original potential $\hat{A}_{(2)}$, but left all other vector potentials (including the Kaluza-Klein vectors) in their original undualised forms.
} 
(in some cases) a dilatonic scalar, are possible. In all cases, we are concerned with the situation where all the Yang-Mills fields of the $S O(n+1)$ isometry group of the $n$-sphere can be included in the reduction Ansatz.

\begin{tabular}{|c|c|c|c|c|c|c|}
\hline$p$-form & Dilaton & Higher-Dim & Lower-Dim. & Sphere & Gauge Group & Extra fields \\
\hline \hline$F_{(2)}$ & Yes & Any $D$ & $D-2$ & $S^{2}$ & $S O(3)$ & None \\
\hline$F_{(3)}$ & Yes & Any $D$ & $D-3$ & $S^{3}$ & $S O(4)$ & $A_{(2)}$ \\
\hline$F_{(3)}$ & Yes & Any $D$ & 3 & $S^{D-3}$ & $S O(D-2)$ & None \\
\hline$F_{(4)}$ & No & 11 & 7 & $S^{4}$ & $S O(5)$ & $A_{(3)}^{i}$ \\
\hline$F_{(4)}$ & No & 11 & 4 & $S^{7}$ & $S O(8)$ & $\phi_{[i j k \ell]_{+}}$ \\
\hline$F_{(5)}=* F_{(5)}$ & No & 10 & 5 & $S^{5}$ & $S O(6)$ & None \\
\hline
\end{tabular}

Table 1: The possible cases for Kaluza-Klein $S^{n}$ reduction with $S O(n+1)$ gauge fields. The last column indicates what additional fields, beyond the metric, the gauge fields $A_{(1)}^{i j}$ and the scalars $T_{i j}$, are massless, and must therefore be included, in a consistent truncation (see discussion in section 1).

\section{Consistent $S^{3}$ reduction}

We start from the bosonic string in $D$ dimensions, with the low-energy effective Lagrangian ${ }^{9}$

$$
\mathcal{L}_{D}=\hat{R} \hat{*} \mathbb{1}-\frac{1}{2} \hat{*} d \hat{\phi} \wedge d \hat{\phi}-\frac{1}{2} e^{-a \hat{\phi}} \hat{*} \hat{F}_{(3)} \wedge \hat{F}_{(3)},
$$

where the positive constant $a$ is given by (77) so that the global symmetry from a $T^{n}$ reduction would be $\mathbb{R} \times O(n, n)$ rather than merely $G L(n, \mathbb{R})$, as discussed in section 2 . As we argued there, we can now conjecture that it should be possible to perform a consistent Kaluza-Klein reduction on $S^{3}$, keeping all the $S O(4)$ Yang-Mills fields $A_{(1)}^{i j}$, together with the scalar fields described by the symmetric tensor $T_{i j}$, where $i$ is a vector under $S O(4)$, and also the 2-form potential $A_{(2)}$.

We find that there is indeed an Ansatz for a consistent Kaluza-Klein reduction on $S^{3}$, given by

$$
d \hat{s}_{D}^{2}=Y^{\frac{1}{D-2}}\left(\Delta^{\frac{2}{D-2}} d s_{D-3}^{2}+g^{-2} \Delta^{-\frac{D-4}{D-2}} T_{i j}^{-1} \mathcal{D} \mu^{i} \mathcal{D} \mu^{j}\right),
$$

\footnotetext{
${ }^{9}$ Later, in section 7 , we shall include the cosmological type term $-\frac{1}{2} m^{2}(D-26) e^{\frac{1}{2} a \hat{\phi}}$ that arises when $D \neq 26$, as a result of the conformal anomaly. For now, we restrict attention to the purely classical Lagrangian for gravity coupled to a 3 -form and a dilaton.
} 


$$
\begin{aligned}
& e^{\sqrt{(D-2) / 2} \hat{\phi}}=\Delta^{-1} Y^{(D-4) / 4} \\
& \hat{F}_{(3)}=F_{(3)}+\frac{1}{6} \epsilon_{i_{1} i_{2} i_{3} i_{4}}\left(g^{-2} U \Delta^{-2} \mathcal{D} \mu^{i_{1}} \wedge \mathcal{D} \mu^{i_{2}} \wedge \mathcal{D} \mu^{i_{3}} \mu^{i_{4}}\right. \\
& \left.\quad-3 g^{-2} \Delta^{-2} D \mu^{i_{1}} \wedge \mathcal{D} \mu^{i_{2}} \wedge \mathcal{D} T_{i_{3} j} T_{i_{4} k} \mu^{j} \mu^{k}-3 g^{-1} \Delta^{-1} F_{(2)}^{i_{1} i_{2}} \wedge \mathcal{D} \mu^{i_{3}} T_{i_{4} j} \mu^{j}\right),
\end{aligned}
$$

where

$$
\begin{aligned}
& \mu^{i} \mu^{i}=1, \quad \Delta=T_{i j} \mu^{i} \mu^{j}, \quad U=2 T_{i k} T_{j k} \mu^{i} \mu^{j}-\Delta T_{i i}, \\
& Y=\operatorname{det}\left(T_{i j}\right)
\end{aligned}
$$

and the indices $i, j, \ldots$ range of 4 values. Here, and in the rest of the paper, a summation over repeated $S O(n+1)$ indices is understood. The gauge-covariant exterior derivative $D$ is defined so that

$$
\mathcal{D} \mu^{i}=d \mu^{i}+g A_{(1)}^{i j} \mu^{j}, \quad \mathcal{D} T_{i j}=d T_{i j}+g A_{(1)}^{i k} T_{k j}+g A_{(1)}^{j k} T_{i k}
$$

where $A_{(1)}^{i j}$ denotes the $S O(4)$ gauge potentials coming from the isometry group of the 3 -sphere, and

$$
F_{(2)}^{i j}=d A_{(1)}^{i j}+g A_{(1)}^{i k} \wedge A_{(1)}^{k j} .
$$

Thus the lower-dimensional fields appearing in the Kaluza-Klein Ansatz comprise the metric $d s_{D-3}^{2}$, the six gauge potentials $A_{(1)}^{i j}$ of $S O(4)$, the ten scalar fields described by the symmetric tensor $T_{i j}$, and the 2 -form potential $A_{(2)}$, whose (Chern-Simons modified) field strength is $F_{(3)}$. The form of the Ansatz that we have obtained here closely parallels the structure found in [18] for the $S^{5}$ reduction of type IIB supergravity.

In order to demonstrate the consistency of the Kaluza-Klein reduction with the above Ansatz, we substitute it into the $D$-dimensional equations of motion 10 that follow from (13). These are

$$
\begin{aligned}
d \hat{*} d \hat{\phi} & =-\frac{1}{2} a e^{-a \phi} \hat{F}_{(3)} \wedge \hat{*} \hat{F}_{(3)}, \\
d\left(e^{-a \phi} \hat{*}_{(3)}\right) & =0 \\
\hat{R}_{M N} & =\frac{1}{2} \partial_{M} \phi \partial_{N} \phi+\frac{1}{4}\left(\hat{F}_{M N}^{2}-\frac{2}{3(D-2)} \hat{F}_{(3)}^{2} \hat{g}_{M N}\right) .
\end{aligned}
$$

\footnotetext{
${ }^{10}$ We shall not consider the reduction of the $D$-dimensional Einstein equation in detail in this paper, on account of its complexity; this will be addressed in future work. In practice, in all cases that have been examined, the Einstein equation seems always to give consistent results provided that the equations of motion for all the other fields are consistent. Furthermore, the agreement of our reduction Anstaz with previously-established special cases provides further supporting evidence for the consistency of the Einstein equation.
} 
In addition, we have the Bianchi identity $d \hat{F}_{(3)}=0$. Taking this first, we find upon substituting $\hat{F}_{(3)}$ from (14) into $d \hat{F}_{(3)}=0$ that the lower-dimensional field $F_{(3)}$ must satisfy the Bianchi identity

$$
d F_{(3)}=\frac{1}{8} \epsilon_{i_{1} i_{2} i_{3} i_{4}} F_{(2)}^{i_{1} i_{2}} \wedge F_{(2)}^{i_{3} i_{4}} .
$$

All other terms arising from $d \hat{F}_{(3)}$ vanish identically. (The calculation is quite involved, and the Schoutens identity $\epsilon_{\left[i_{1} i_{2} i_{3} i_{4}\right.} V_{\left.i_{5}\right]} \equiv 0$ is useful.)

In order to substitute the Ansatz into the field equation for $\hat{F}_{(3)}$, we must first calculate the Hodge dual of $\hat{F}_{(3)}$ given in (14). This is a straightforward, although somewhat involved calculation, and we find

$$
\begin{aligned}
e^{-\sqrt{8 /(D-2)} \hat{\phi}} \hat{*} \hat{F}_{(3)}= & \frac{1}{6 g^{3}} \epsilon_{i j k \ell} Y^{-1} * F_{(3)} \wedge \mu^{i} \mathcal{D} \mu^{j} \wedge \mathcal{D} \mu^{k} \wedge \mathcal{D} \mu^{\ell}-g U \epsilon_{D-3} \\
& +g^{-1} T_{i j}^{-1} * \mathcal{D} T_{j k} \wedge\left(\mu^{k} \mathcal{D} \mu^{i}\right)-\frac{1}{2 g^{2}} T_{i k}^{-1} T_{j l}^{-1} * F_{(2)}^{i j} \wedge \mathcal{D} \mu^{k} \wedge \mathcal{D} \mu^{\ell} .
\end{aligned}
$$

Substituting into (18), with $a$ given by (7), we (eventually) read off the lower-dimensional equations of motion

$$
\begin{aligned}
& (-1)^{D} \mathcal{D}\left(T_{i k}^{-1} T_{j \ell}^{-1} * F_{(2)}^{k \ell}\right)=-2 g T_{k[i}^{-1} * \mathcal{D} T_{j] k}-\frac{1}{2} \epsilon_{i j k \ell} Y^{-1} * F_{(3)} \wedge F_{(2)}^{k \ell}, \\
& (-1)^{D} \mathcal{D}\left(\widetilde{T}_{i k}^{-1} * \mathcal{D} \widetilde{T}_{k j}\right)=2 g^{2}\left[2 T_{i k} T_{j k}-T_{i j} T_{k k}\right] \epsilon_{D-3}-T_{\ell m}^{-1} T_{i k}^{-1} * F_{(2)}^{\ell k} \wedge F_{(2)}^{m j} \\
& -\frac{1}{4} \delta_{i j}\left(2 g^{2}\left[2 T_{n k} T_{n k}-\left(T_{k k}\right)^{2}\right] \epsilon_{D-3}-T_{\ell m}^{-1} T_{n k}^{-1} * F_{(2)}^{\ell k} \wedge F_{(2)}^{m n}\right), \\
& d\left(Y^{-1} * F_{(3)}\right)=0
\end{aligned}
$$

We have introduced the unimodular matrix $\widetilde{T}_{i j}$, constructed from $T_{i j}$ by extracting the determinant factor $Y$ (see 15$)$,

$$
\widetilde{T}_{i j}=Y^{\frac{1}{4}} T_{i j}
$$

Again, there are many other terms that arise from acting with the exterior derivative that cancel amongst themselves, after making use of the Schoutens identity. The consistency of the reduction Ansatz manifests itself in the remarkable fact that one reads off consistent lower-dimensional equations of motion in which all the dependence on the internal $S^{3}$ coordinates $\mu^{i}$ has cancelled.

Next, we consider the equation of motion for the dilaton $\hat{\phi}$. From (14) we find

$$
d \hat{\phi}=\sqrt{\frac{2}{D-2}}\left(\frac{1}{4}(D-4) Y^{-1} * d Y-\Delta^{-1} d \Delta\right) .
$$

Since $\Delta$ has dependence on the $S^{3}$ coordinates $\mu^{i}$ one of the terms arising here will involve the quantity

$$
T_{i j} \mu^{i} \mathcal{D} \mu^{j}
$$


It is therefore necessary to evaluate the Hodge dual of this 1-form; we find

$$
\hat{*}\left(T_{i j} \mu^{i} \mathcal{D} \mu^{j}\right)=-\frac{1}{2} \epsilon_{i_{1} i_{2} i_{3} i_{4}} T_{i \ell} \mu^{i} \epsilon_{D-3} \wedge\left(\Delta T_{i_{1} \ell}-T_{i_{1} j} T_{k \ell} \mu^{j} \mu^{k}\right) \mu^{i_{2}} \mathcal{D} \mu^{i_{3}} \wedge \mathcal{D} \mu^{i_{4}} .
$$

After some involved manipulations, we find that the $D$-dimensional dilaton equation of motion in (18) implies that $Y$ satisfies

$$
\begin{aligned}
\frac{D-5}{4}(-1)^{D} d\left(Y^{-1} * d Y\right)= & \frac{1}{2} g^{2}\left(2 T_{i j} T_{i j}-\left(T_{i i}\right)^{2}\right) \epsilon_{D-3}-Y^{-1} * F_{(3)} \wedge F_{(3)} \\
& -\frac{1}{4} Y^{-1} T_{i k}^{-1} T_{j \ell}^{-1} * F_{(2)}^{i j} \wedge F_{(2)}^{k \ell}
\end{aligned}
$$

The full set of $(D-3)$-dimensional equations of motion can be derived from the Lagrangian

$$
\begin{aligned}
\mathcal{L}_{D-3}= & R * \mathbb{1}-\frac{D-5}{16} Y^{-2} * d Y \wedge d Y-\frac{1}{4} \widetilde{T}_{i j}^{-1} * \mathcal{D} \widetilde{T}_{j k} \wedge \widetilde{T}_{k \ell}^{-1} \mathcal{D} \widetilde{T}_{\ell i} \\
& -\frac{1}{2} Y^{-1} * F_{(3)} \wedge F_{(3)}-\frac{1}{4} Y^{-\frac{1}{2}} \widetilde{T}_{i k}^{-1} \widetilde{T}_{j \ell}^{-1} * F_{(2)}^{i j} \wedge F_{(2)}^{k \ell}-V * \mathbb{1},
\end{aligned}
$$

where the potential $V$ is given by

$$
V=\frac{1}{2} g^{2} Y^{\frac{1}{2}}\left(2 \widetilde{T}_{i j} \widetilde{T}_{i j}-\left(\widetilde{T}_{i i}\right)^{2}\right) .
$$

The 3 -form field strength $F_{3}$ is given by

$$
F_{(3)}=d A_{(2)}+\frac{1}{8} \epsilon_{i j k \ell}\left(F_{(2)}^{i j} \wedge A_{(1)}^{k \ell}-\frac{1}{3} g A_{(1)}^{i j} \wedge A_{(1)}^{k m} \wedge A_{(1)}^{m \ell}\right),
$$

which implies that $F_{(3)}$ satisfies the Bianchi identity (19).

\section{Truncations to previous results}

In this section, we consider two truncations of the $S^{3}$ Kaluza-Klein reduction of the bosonic string that we constructed in the previous section.

\subsection{Truncation from $S O(4)$ to $S U(2)$}

The first truncation turns the reduction into a "standard" one, for which the consistency becomes immediately understandable from group-theoretic arguments. Specifically, we may truncate the $S O(4)$ Yang-Mills gauge fields that arise from the $S^{3}$ reduction to a set of $S U(2)$ gauge fields, corresponding either to the left-action, or to the right-action, of $S U(2)$ on the $S^{3} \sim S U(2)$ group manifold. This is achieved by imposing a self-dual or anti-self-dual truncation on the original $S O(4)$ gauge potentials $A_{(1)}^{i j}$,

$$
A_{(1)}^{i j}= \pm \frac{1}{2} \epsilon_{i j k \ell} A_{(1)}^{k \ell} .
$$


The choice of sign governs whether we are retaining the gauge fields of $S U(2)_{L}$ or of $S U(2)_{R}$ in the truncation of $S O(4) \sim S U(2)_{L} \times S U(2)_{R}$. The two choices are equivalent, up to convention choices, and we shall pick the plus sign in (30) for definiteness. It is convenient to take the $i, j, \ldots$ indices to range over the values $0,1,2,3$, and to write the remaining gauge potentials in terms of the $S U(2)$ triplet $A_{(1)}^{\alpha}$, with

$$
A_{(1)}^{01}=A_{(1)}^{23} \equiv \frac{1}{2} A_{(1)}^{1}, \quad A_{(1)}^{02}=A_{(1)}^{31} \equiv-\frac{1}{2} A_{(1)}^{2}, \quad A_{(1)}^{03}=A_{(1)}^{12} \equiv \frac{1}{2} A_{(1)}^{3} .
$$

These are the gauge fields of $S U(2)_{L}$.

At the same time as we impose the self-dual truncation (30) on the gauge potentials, we must also truncate the scalar fields $T_{i j}$, in order to be consistent with the equations of motion for the truncated gauge fields. In fact we should retain just a single scalar degree of freedom $X$, so that $T_{i j}$ becomes

$$
T_{i j}=X \delta_{i j}
$$

Note that from (15) we shall now have $Y=X^{4}$. It is convenient also to give an explicit parametrisation of the $\mu^{i}$ coordinates, in terms of Euler angles on $S^{3}$ :

$$
\mu_{0}+\mathrm{i} \mu_{3}=\cos \frac{1}{2} \theta e^{\mathrm{i}(\psi+\phi) / 2}, \quad \mu_{1}+\mathrm{i} \mu_{2}=\sin \frac{1}{2} \theta e^{\mathrm{i}(\psi-\phi) / 2} .
$$

In terms of these we can then define the left-invariant 1-forms $\sigma_{\alpha}$ on $S^{3}$, according to

$$
\sigma_{1}+\mathrm{i} \sigma_{2}=e^{-\mathrm{i} \psi}(d \theta+\mathrm{i} \sin \theta d \phi), \quad \sigma_{3}=d \psi+\cos \theta d \phi
$$

These satisfy the $S U(2)$ algebra $d \sigma_{\alpha}=-\frac{1}{2} \epsilon_{\alpha \beta \gamma} \sigma_{\beta} \wedge \sigma_{\gamma}$.

With these preliminaries, we can now present our results for the reduction Anstaz for this $S U(2)$ truncation of the original $S O(4)$ Kaluza-Klein reduction. We find that the metric, dilaton and 3-form Ansätze given in (14)-(14) reduce to

$$
\begin{aligned}
d \hat{s}_{D}^{2} & =X^{\frac{6}{D-2}} d s_{D-3}^{2}+\frac{1}{4} X^{-\frac{2(D-5)}{D-2}} \sum_{\alpha}\left(\sigma_{\alpha}-g A_{(1)}^{\alpha}\right)^{2}, \\
e^{\sqrt{(D-2) / 2} \hat{\phi}} & =X^{D-5}, \\
\hat{F}_{(3)} & =F_{(3)}-\frac{1}{4 g^{2}} \Omega_{(3)}-\frac{1}{12 g} \epsilon_{\alpha \beta \gamma} F_{(2)}^{\alpha} \wedge\left(\sigma_{\beta}-g A_{(1)}^{\beta}\right) \wedge\left(\sigma_{\gamma}-g A_{(1)}^{\gamma}\right),
\end{aligned}
$$

where

$$
\Omega_{(3)} \equiv \frac{1}{6} \epsilon_{\alpha \beta \gamma}\left(\sigma_{\alpha}-g A_{(1)}^{\alpha}\right) \wedge\left(\sigma_{\beta}-g A_{(1)}^{\beta}\right) \wedge\left(\sigma_{\gamma}-g A_{(1)}^{\gamma}\right)
$$

is the volume form on the 3 -sphere.

It is easy to verify that this $S U(2)$ truncation of the full $S O(4)$ reduction Ansatz of section 2 is a consistent one. As we remarked above, there is no longer anything "surprising" about the consistency in this case, since the truncation has set to zero all fields that 
transformed non-trivially under $S U(2)_{R}$. In other words, the $S U(2)_{L}$ Ansatz in this section retains all the singlets under $S U(2)_{R}$, while discarding all the non-singlets. Such a truncation is necessarily consistent, since non-linear products of the fields that are retained can never generate non-singlets under $S U(2)_{R}$. A related point is that the fields that remain in the reduction Ansatz parameterise homogeneous deformations of the 3-sphere. A particular case of this $S U(2)$ reduction has appeared previously in the literature, in the $S^{3}$ reduction of $N=1$ supergravity from $D=10$ to $D=7$ [29].

\subsection{Truncation from $S O(4)$ to $U(1) \times U(1)$}

The second truncation that we shall consider here involves retaining only the $U(1) \times U(1)$ subgroup of the original $S U(2) \times S U(2)$ gauged fields of the full $S O(4)$ reduction Ansatz of section 2. It is convenient now to take the $S O(4)$ indices $i, j, \ldots$ to range over the values $1,2,3,4$. The truncation amounts to setting all gauge potentials $A_{(1)}^{i j}$ to zero except for $A_{(1)}^{12}$ and $A_{(1)}^{34}$, for which we write

$$
A_{(1)}^{12} \equiv A_{(1)}^{1}, \quad A_{(1)}^{34} \equiv A_{(1)}^{2}
$$

It is also convenient now to parameterise the coordinates $\mu^{i}$ on $S^{3}$ as

$$
\mu_{1}+\mathrm{i} \mu_{2}=\tilde{\mu}_{1} e^{\mathrm{i} \phi_{1}}, \quad \mu_{3}+\mathrm{i} \mu_{4}=\tilde{\mu}_{2} e^{\mathrm{i} \phi_{2}} .
$$

At the same time as making the truncation of the gauge fields, consistency with their equations of motion requires that we set certain of the scalar fields to zero, so that what remains is just two scalars $X_{1}$ and $X_{2}$ as follows:

$$
T_{i j}=\operatorname{diag}\left(X_{1}, X_{1}, X_{2}, X_{2}\right)
$$

Note that we shall now have

$$
Y=\left(X_{1} X_{2}\right)^{2}, \quad \Delta=X_{1} \tilde{\mu}_{1}^{2}+X_{2} \tilde{\mu}_{2}^{2}, \quad U=2 \sum_{i=1}^{2}\left(X_{i}^{2} \tilde{\mu}_{i}^{2}-\Delta X_{i}\right) .
$$

After substituting the above truncation and reparametrisation into the original KaluzaKlein Ansätze in section 2, we find that the metric and dilaton Asätze become

$$
\begin{aligned}
d \hat{s}_{D}^{2}= & \left(X_{1} X_{2}\right)^{\frac{2}{D-2}}\left(\Delta^{\frac{2}{D-2}} d s_{D-3}^{2}\right. \\
& \left.+g^{-2} \Delta^{-\frac{D-4}{D-2}} \sum_{i=1}^{2} X_{i}^{-1}\left(d \tilde{\mu}_{i}^{2}+\tilde{\mu}_{i}^{2}\left(d \phi_{i}-g A_{(1)}^{i}\right)^{2}\right)\right) \\
e^{\sqrt{(D-2) / 2} \hat{\phi}}= & \Delta^{-1}\left(X_{1} X_{2}\right)^{\frac{D-4}{2}}
\end{aligned}
$$


The Ansatz for the 3-form field $\hat{F}_{(3)}$ in this $U(1)^{2}$ truncation is most simply expressed in terms of the expression for the dual of $\hat{F}_{(3)}$. Making the truncation in (20), we find

$$
\begin{aligned}
e^{-\sqrt{8 /(D-2)} \hat{\phi}} \hat{*} \hat{F}_{(3)}= & -2 g \sum_{i=1}^{2}\left(X_{i}^{2} \tilde{\mu}_{i}^{2}-\Delta X_{i}\right) \epsilon_{D-3}+\frac{1}{2 g} \sum_{i=1}^{2} X_{i}^{-1} * d X_{i} \wedge d\left(\tilde{\mu}_{i}^{2}\right) \\
& -\frac{1}{2 g^{2}} \sum_{i=1}^{2} X_{i}^{-2} d\left(\tilde{\mu}_{i}^{2}\right) \wedge\left(d \phi_{i}-g A_{(1)}^{i}\right) \wedge * F_{(2)}^{i}+g^{-3} Y^{-1} * F_{(3)}
\end{aligned}
$$

The Ansatz for $\hat{F}_{(3)}$ itself is also easily obtainable by imposing the $U(1)^{2}$ truncation on the general $S O(4)$ Ansatz (14).

Note that in the $U(1)^{2}$ truncation the question of the consistency of the reduction is still a non-trivial one, since the two scalars $X_{1}$ and $X_{2}$ parameterise inhomogeneous deformations of the 3 -sphere. Of course since we have already argued that the $S O(4)$ reduction in section 2 is consistent, the consistency for the $U(1)^{2}$ truncation is a consequence.

A particular case of this $U(1)^{2}$ truncation appeared previously in the literature [30], where it was obtained for the case $D=10$ by taking a singular limit of the $S^{4}$ reduction of eleven-dimensional supergravity that was constructed in [0].

\section{$5 S^{D-3}$ reduction and $D=3, N=8$ gauged supergravity}

As we discussed in section 2, it is natural to conjecture that the theory of gravity coupled to a dilaton and a 3 -form, described by (13) with $a$ given by (7), should also admit a consistent reduction to three dimensions on the sphere $S^{D-3}$, in which all the Yang-Mills gauge fields of $S O(D-2)$ are retained. Additionally, we should keep the $\frac{1}{2}(D-1)(D-2)$ scalar fields described by the symmetric tensor $T_{i j}$, where $i$ is a vector index of $S O(D-2)$. We find that indeed such a consistent reduction is possible, and that the Kaluza-Klein Ansatz is given by

$$
\begin{aligned}
d \hat{s}_{D}^{2}= & Y^{\frac{1}{D-2}}\left(\Delta^{\frac{D-4}{D-2}} d s_{3}^{2}+g^{-2} \Delta^{-\frac{2}{D-2}} T_{i j}^{-1} \mathcal{D} \mu^{i} \mathcal{D} \mu^{j}\right), \\
e^{-\sqrt{(D-2) / 2} \hat{\phi}=} & \Delta^{-1} Y^{1 / 2}, \\
\hat{F}_{(3)}= & -g U \epsilon_{3}+g^{-1} T_{i j}^{-1} * \mathcal{D} T_{j k} \wedge\left(\mu^{k} \mathcal{D} \mu^{i}\right) \\
& -\frac{1}{2 g^{2}} T_{i k}^{-1} T_{j l}^{-1} * F_{(2)}^{i j} \wedge \mathcal{D} \mu^{k} \wedge \mathcal{D} \mu^{\ell},
\end{aligned}
$$

where the various quantities appearing here are again given in (15), (16) and (17), but now the indices $i, j, \ldots$ range over $(D-2)$ values. Thus the field content in Kaluza-Klein reduced three-dimensional theory comprises the metric $d s_{3}^{2}$, the $\frac{1}{2}(D-2)(D-3)$ gauge potentials 
$A_{(1)}^{i j}$ of $S O(D-2)$, and the $\frac{1}{2}(D-1)(D-2)$ scalars described by the symmetric tensor $T_{i j}$. The calculation of the Hodge dual of the 3 -form $\hat{F}_{(3)}$ is again a mechanical, although involved, calculation. We find that it is given by

$$
\begin{aligned}
e^{-\sqrt{8 /(D-2)} \hat{\phi}} \hat{*} \hat{F}_{(3)}= & \frac{g^{-(D-4)}}{(D-3) !} \epsilon_{i_{1} \cdots i_{D-2}}\left(g U \Delta^{-2} \mu^{i_{1}} \mathcal{D} \mu^{i_{2}} \cdots \mathcal{D} \mu^{i_{D-2}}\right. \\
& -(D-3) \Delta^{-2} T_{i_{1} j} \mathcal{D} T_{i_{2} k} \mathcal{D} \mu^{i_{3}} \cdots \mathcal{D} \mu^{i_{D}-2} \mu^{j} \mu^{k} \\
& \left.-\frac{(D-3)(D-4)}{2} F_{(2)}^{i_{1} i_{2}} T_{i_{3} j} \mathcal{D} \mu^{i_{4}} \mathcal{D} \mu^{i_{D-2}} \mu^{j}\right)
\end{aligned}
$$

where we have suppressed the wedge symbols in products of differential forms in order to economise on space.

It is again a straightforward, although lengthy, procedure to substitute the above Ansatz into the $D$-dimensional equations of motion (18), and to verify that there is a consistent reduction to equations of motion for the three-dimensional fields. We find that these equations can be derived from the following three-dimensional Lagrangian:

$$
\begin{aligned}
\mathcal{L}_{3}= & R * \mathbb{1}-\frac{1}{4(D-2)} Y^{-2} * d Y \wedge d Y-\frac{1}{4} \widetilde{T}_{i j}^{-1} * \mathcal{D} \widetilde{T}_{j k} \wedge \widetilde{T}_{k \ell}^{-1} \mathcal{D} \widetilde{T}_{\ell i} \\
& -\frac{1}{4} Y^{-\frac{2}{D-2}} \widetilde{T}_{i k}^{-1} \widetilde{T}_{j \ell}^{-1} * F_{(2)}^{i j} \wedge F_{(2)}^{k \ell}-V * \mathbb{1},
\end{aligned}
$$

where $Y=\operatorname{det}\left(T_{i j}\right)$, and $T_{i j}$ is written in terms of the unimodular $(D-2) \times(D-2)$ matrix $\widetilde{T}_{i j}$ as $T_{i j}=Y^{1 /(D-2)} \widetilde{T}_{i j}$. The potential $V$ is given by

$$
V=\frac{1}{2} g^{2} Y^{\frac{2}{D-2}}\left(2 \widetilde{T}_{i j} \widetilde{T}_{i j}-\left(\widetilde{T}_{i i}\right)^{2}\right)
$$

An application of this dimensional reduction that is of particular interest arises if we take $D=10$, since then the higher-dimensional starting point will be the bosonic sector of $N=1$ supergravity in ten dimensions. The reduction on $S^{7}$ then yields a three-dimensional theory that is the bosonic sector of an $S O(8)$ gauged supergravity, with $N=8$ (i.e. half of maximal) supersymmetry. As well as the 28 gauge fields, there are in total 36 scalars, described by the unimodular symmetric tensor $\widetilde{T}_{i j}$ and the scalar $Y$. These transform as a 35 and a 1 under $S O(8)$, respectively. Evidently, if we reduced the full $N=1$ theory in $D=10$, including the fermions, we would obtain $N=8$ gauged $S O(8)$ supergravity in three dimensions. This appears to be the first example of such a gauged supergravity in $D=3$. Previous examples of gauged three-dimensional supergravities in the literature have been of the type constructed in [31], with $S O(p) \times S O(q)$ gauge fields and a pure cosmological constant term implying the existence of an $\mathrm{AdS}_{3}$ ground-state solution. In fact there are no scalar fields, and hence no scalar potential, in the theories constructed in [31]. By contrast, the gauged supergravity that we have obtained here has 36 scalars with the potential (49). 
The theory does not admit an $\mathrm{AdS}_{3}$ solution, but it may allow domain-wall solutions that preserve half of the supersymmetry.

\section{$6 \quad S^{2}$ reduction}

Here, we construct the Kaluza-Klein Ansatz for the reduction of (3) with $p=2$ and $a$ given by (6). Thus our starting point is

$$
\mathcal{L}_{D}=\hat{R} \hat{*} \mathbb{1}-\frac{1}{2} \hat{*} d \hat{\phi} \wedge d \hat{\phi}-\frac{1}{2} e^{-a \hat{\phi}} \hat{*} \hat{F}_{(2)} \wedge \hat{F}_{(2)},
$$

where the positive constant $a$ is given by (66). From (50) we derive the equations of motion

$$
\begin{aligned}
d \hat{*} d \hat{\phi} & =\frac{1}{2}(-1)^{D} a e^{-a \hat{\phi}} \hat{*} \hat{F}_{(2)} \wedge \hat{F}_{(2)}, \\
d\left(e^{\left.-a \hat{\phi}_{\hat{*}} \hat{F}_{(2)}\right)}\right. & =0 \\
\hat{R}_{M N} & =\frac{1}{2} \partial_{M} \hat{\phi} \partial_{N} \hat{\phi}+\frac{1}{2} e^{-a \hat{\phi}}\left(\hat{F}_{M N}^{1}-\frac{1}{2(D-2)} \hat{F}_{(2)}^{2} \hat{g}_{M N}\right) .
\end{aligned}
$$

We find that there is a consistent reduction Ansatz on $S^{2}$, given by

$$
\begin{aligned}
d \hat{s}_{D}^{2}= & Y^{\frac{1}{D-2}}\left(\Delta^{\frac{1}{D-2}} d s_{D-2}^{2}+g^{-2} \Delta^{-\frac{D-3}{D-2}} T_{i j}^{-1} \mathcal{D} \mu^{i} \mathcal{D} \mu^{j}\right) \\
e^{\sqrt{\frac{2(D-2)}{D-1}} \hat{\phi}=} & \Delta^{-1} Y^{\frac{D-3}{D-1}} \\
\hat{F}_{(2)}= & \frac{1}{2} \epsilon_{i j k}\left(g^{-1} U \Delta^{-2} \mu^{i} \mathcal{D} \mu^{j} \wedge \mathcal{D} \mu^{k}-2 g^{-1} \Delta^{-2} \mathcal{D} \mu^{i} \wedge \mathcal{D} T_{j \ell} T_{k m} \mu^{\ell} \mu^{m}\right. \\
& \left.-\Delta^{-1} F_{(2)}^{i j} T_{k \ell} \mu^{\ell}\right) .
\end{aligned}
$$

Again, the various quantities appearing here are given in (15), (16) and (17), but with the indices $i, j, \ldots$ ranging over 3 values. The dual of the 2 -form then turns out to be given by

$$
\begin{aligned}
e^{-\sqrt{\frac{2(D-1)}{D-2}} \hat{\phi}} \hat{*}_{(2)}= & -g U \epsilon_{D-2}+g^{-1} T_{i j}^{-1} * \mathcal{D} T_{j k} \wedge\left(\mu^{k} \mathcal{D} \mu^{i}\right) \\
& -\frac{1}{2 g^{2}} T_{i k}^{-1} T_{j l}^{-1} * F_{(2)}^{i j} \wedge \mathcal{D} \mu^{k} \wedge \mathcal{D} \mu^{\ell}
\end{aligned}
$$

The field content of the Kaluza-Klein reduced theory comprises the $(D-2)$-dimensional

metric $d s_{D-2}^{2}$, the three gauge potentials $A_{(1)}^{i j}$ of $S O(3)$, and the six scalar fields $T_{i j}$.

Substituting the Ansatz into the $D$-dimensional equations of motion (51), we find that it yields a consistent Kaluza-Klein $S^{2}$ reduction, with the $(D-2)$-dimensional fields satisfying equations of motion that follow from the Lagrangian

$$
\begin{aligned}
\mathcal{L}_{D-2}= & R * \mathbb{1}-\frac{D-4}{3(D-1)} Y^{-2} * d Y \wedge d Y-\frac{1}{4} \widetilde{T}_{i j}^{-1} * \mathcal{D} \widetilde{T}_{j k} \wedge \widetilde{T}_{k \ell}^{-1} \mathcal{D} \widetilde{T}_{\ell i} \\
& -\frac{1}{4} Y^{-\frac{2}{3}} \widetilde{T}_{i k}^{-1} \widetilde{T}_{j \ell}^{-1} * F_{(2)}^{i j} \wedge F_{(2)}^{k \ell}-V * \mathbb{1},
\end{aligned}
$$


where $Y=\operatorname{det}\left(T_{i j}\right)$, and $T_{i j}$ is written in terms of the unimodular $3 \times 3$ matrix $\widetilde{T}_{i j}$ as $T_{i j}=Y^{1 / 3} \widetilde{T}_{i j}$. The potential $V$ is given by

$$
V=\frac{1}{2} g^{2} Y^{\frac{2}{3}}\left(2 \widetilde{T}_{i j} \widetilde{T}_{i j}-\left(\widetilde{T}_{i i}\right)^{2}\right)
$$

In view of our earlier observation that the $D$-dimensional Lagrangian (50), with the constant $a$ given by (6), can itself be thought of as an ordinary $S^{1}$ Kaluza-Klein reduction of pure gravity in $(D+1)$ dimensions, it follows that we can also interpret our result as a consistent reduction of $(D+1)$-dimensional pure gravity. The internal space is not simply $S^{1} \times S^{2}$, however, since the 2 -form field $F_{(2)}$ in $D$ dimensions, which is the Kaluza-Klein vector of the $S^{1}$ reduction from $(D+1)$ dimensions, is topologically non-trivial. One can see from (55) that if, for example, the scalars were all taking trivial values, the 2 -form field $\hat{F}_{(2)}$ would be just the volume-form on $S^{2}$ (like in a Dirac monopole configuration). Thus the reduction from $(D+1)$ dimensions is actually on a manifold that is topologically $S^{3}$. In fact we can easily lift the metric Ansatz in (53) to give the Ansatz for the reduction from $(D+1)$ dimensions, by incorporating the standard $S^{1}$ reduction step

$$
d \hat{s}_{D+1}^{2}=e^{2 \alpha \hat{\phi}} d \hat{s}_{D}^{2}+e^{-2 \alpha(D-2) \hat{\phi}}\left(d z+\hat{A}_{(1)}\right)^{2},
$$

where $\hat{F}_{(2)}=d \hat{A}_{(1)}$, and the fields on the right-hand side are given in (53)-(55). Thus we find

$$
d \hat{s}_{D+1}^{2}=Y^{\frac{2}{D-1}} d s_{D-2}^{2}+\Delta^{-1} Y^{\frac{2}{D-1}} T_{i j}^{-1} \mathcal{D} \mu^{i} \mathcal{D} \mu^{j}+\Delta Y^{-\frac{D-3}{D-1}}\left(d z+\hat{A}_{(1)}\right)^{2}
$$

This is an unusual type of $S^{3}$ reduction, in which the three $S O(3)$ Yang-Mills fields $A_{(1)}^{i j}$ and the six scalar fields $T_{i j}$ parameterise inhomogeneous deformations of the 3 -sphere.

\section{Conformal anomaly terms}

Until now we have focussed our attention on the purely classical theories of gravity coupled to a $p$-form field strength and a dilaton. One of the two cases that admits consistent sphere reductions turned out to be when $p=3$, and in fact the Lagrangian (13) is precisely the leading-order expression for the low-energy limit of the $D$-dimensional bosonic string. Of course the bosonic string suffers from a conformal anomaly if the dimension $D$ is not equal to 26. It turns out that the effect of this anomaly is to generate an additional term in the effective action [33], which vanishes at $D=26$, so that (13) is replaced by

$$
\mathcal{L}_{D}=\hat{R} \hat{*} \mathbb{1}-\frac{1}{2} \hat{*} d \hat{\phi} \wedge d \hat{\phi}-\frac{1}{2} e^{-a \hat{\phi}} \hat{*} \hat{F}_{(3)} \wedge \hat{F}_{(3)}-\frac{1}{2} m^{2}(D-26) e^{\frac{1}{2} a \hat{\phi}} \hat{*} \mathbb{1} .
$$


We shall refer to this extra contribution as a "cosmological term." Note that if we were instead considering the theory of gravity, 3-form and dilaton as coming from the low-energy effective theory of the superstring, the $(D-26)$ factor would be replaced by $(D-10)$. In all subsequent discussions in this section, 26 can accordingly be replaced by 10 in the context of the superstring.

It is of interest to see what happens to the previous Kaluza-Klein reductions on $S^{3}$ and $S^{D-3}$ after this extra term is included. We find that the previous $S^{3}$ reduction Ansatz continues to give a consistent reduction, in which all the dependence on the $S^{3}$ coordinates cancels out when it is substituted into the $D$-dimensional equations of motion following from (61). We find that the reduced $(D-3)$-dimensional theory is described by the same Lagrangian (27), but now the potential $V$ given in (28) is replaced by

$$
V=\frac{1}{2} g^{2} Y^{\frac{1}{2}}\left(2 \widetilde{T}_{i j} \widetilde{T}_{i j}-\left(\widetilde{T}_{i i}\right)^{2}\right)+\frac{1}{2} m^{2}(D-26) Y^{\frac{1}{2}} .
$$

The fact that the $S^{3}$ reduction continues to be a consistent one after the inclusion of the cosmological term in (61) could in fact have been foreseen by considering the grouptheory arguments that we developed in section 2. In the absence of the cosmological term, we observed that the global symmetry group after a $T^{3}$ reduction is $\mathbb{R} \times O(3,3)$, which is large enough to contain $O(3) \times O(3)$ as a compact subgroup, and hence to permit an $S O(4)$ gauging. The inclusion of the cosmological term in (61) breaks the $\mathbb{R}$ factor in the global symmetry, but the $O(3,3)$ factor survives, 1 and so the cosmological term does not present any obstacle to the $S O(4)$ gauging in $D-3$ dimensions.

It is interesting to note that if $D>26$ (or $D>10$ in the case of a supersymmetric string), the potential (62) admits a symmetrical ground-state solution in which all the scalar fields are constant. To see this, we note that for such a solution we must have

$$
\frac{\partial V}{\partial Y}=0, \quad \frac{\partial V}{\partial \widetilde{T}_{i j}}-\frac{1}{4} \delta_{i j} \delta_{k \ell} \frac{\partial V}{\partial \widetilde{T}_{k \ell}}=0 .
$$

(The trace subtraction in the second equation arises because $\widetilde{T}_{i j}$ has unit determinant.) Thus the conditions for a solution with constant scalars imply

$$
V=0, \quad \widetilde{T}_{i j}=\frac{1}{4} \widetilde{T}_{k k} \delta_{i j}
$$

and hence since $\widetilde{T}_{i j}$ is unimodular we must have $\widetilde{T}_{i j}=\delta_{i j}$, and

$$
g=m \sqrt{\frac{D-26}{8}}
$$

\footnotetext{
${ }^{11}$ This can be seen from the fact that the dilaton vector for the cosmological term after the $T^{3}$ reduction is orthogonal to the dilaton vectors that form the positive roots of $O(3,3)$.
} 
with $Y$ arbitrary. Note in particular that the vanishing of $V$ implies that the $(D-3)$ dimensional Einstein equation has no cosmological term, and so it admits Minkowski spacetime as a ground-state solution. One can also find non-trivial solutions that are asymptotically flat.

If we now consider instead the $S^{D-3}$ reduction of the new theory (61), we find that the previously consistent reduction is spoiled by the presence of the additional cosmological term. In particular, it turns out that there is a mis-match between the $S^{D-3}$ dependence from the extra term $e^{\frac{1}{2} a \hat{\phi}} \hat{*} \mathbb{1}$, in comparison to the previous terms, in the $D$-dimensional equation of motion for the dilaton $\hat{\phi}$. Actually, this is not too surprising. It can be understood from the fact that the presence of the cosmological term in (61) breaks the enhanced $O(D-2, D-2)$ global symmetry that occurred previously under a dimensional reduction on $T^{D-3}$, and so there will no longer be an $S O(D-2)$ compact subgroup of the global symmetry group that could permit an $S O(D-2)$ gauging in three dimensions. This can be seen from the fact that the dilaton vector for the cosmological term in (61), after toroidal reduction on $T^{D-3}$, is not orthogonal to the positive root vectors of $O(D-2, D-2)$.

Finally, we may also consider the possible inclusion of an analogous cosmological term in the Lagrangian (50) for gravity, the dilaton and a 2-form field strength. In this case there would not be any direct motivation from bosonic string theory for the inclusion of such a term, but it is nevertheless of interest to see what the effect would be. Thus we may consider whether we may modify the Lagrangian (50) to

$$
\mathcal{L}_{D}=\hat{R} \hat{*} \mathbb{1}-\frac{1}{2} \hat{*} d \hat{\phi} \wedge d \hat{\phi}-\frac{1}{2} e^{-a \hat{\phi}} \hat{*} \hat{F}_{(2)} \wedge \hat{F}_{(2)}-\frac{1}{2} m^{2} e^{b \hat{\phi}} \hat{*} \mathbb{1}
$$

where the dilaton coupling constant $b$ in the cosmological term is chosen so as to maintain the consistency of the Kaluza-Klein $S^{2}$ reduction. It turns out that this is indeed possible, and consistency is achieved if $b$ is the positive constant given by

$$
b^{2}=\frac{2}{(D-1)(D-2)} .
$$

The resulting Kaluza-Klein theory in $(D-2)$ dimensions is described by the Lagrangian (57), but with the potential (58) replaced by

$$
V=\frac{1}{2} g^{2} Y^{\frac{2}{3}}\left(2 \widetilde{T}_{i j} \widetilde{T}_{i j}-\left(\widetilde{T}_{i i}\right)^{2}\right)+\frac{1}{2} m^{2} Y^{\frac{2}{D-2}} .
$$

Again, one could have foreseen the continued consistency of the $S^{2}$ reduction from the fact that if the theory (66) is reduced instead on $T^{2}$, there is still a sufficient enhancement of the global symmetry to permit an $S O(3)$ gauging. Previously, for (50), the generic $G L(2, \mathbb{R})$ 
symmetry was enhanced to to $G L(3, \mathbb{R})$. Now, with the inclusion of the cosmological term in $(66)$, the $\mathbb{R}$ factor in the $G L(3, \mathbb{R})$ is broken, but the $S L(3, \mathbb{R})$ factor remains, and so the compact $S O(3)$ subgroup is still available for the gauging. We can also understand this as follows. Recalling that the original Lagrangian (50) can itself be viewed as a standard $S^{1}$ reduction of pure gravity in $(D+1)$ dimensions, we now observe that the enlarged Lagrangian (66), with $b$ given by (67), is nothing but the $S^{1}$ reduction of the $(D+1)$-dimensional theory of pure gravity with a cosmological constant:

$$
\mathcal{L}_{D+1}=\hat{R}_{D+1} \hat{*} \mathbb{1}-\frac{1}{2} m^{2} \hat{*} \mathbb{1} .
$$

It is then evident that the dimensional reduction of (66) on $T^{2}$ will give the same theory as the dimensional reduction of (69) on $T^{3}$, and so in particular there will be a $S L(3, \mathbb{R})$ global symmetry. ए2

One can again look for solutions of the reduced theory in which all the scalars are constant. The equations of motion following from (68) then imply that

$$
\widetilde{T}_{i j}=\delta_{i j}, \quad Y^{\frac{2(D-5)}{D-2}}=\frac{m^{2}}{g^{2}(D-2)} .
$$

Substituting these back into the potential, we find that at this extremum it is give by

$$
V=\frac{1}{2} m^{2}\left(\frac{D-5}{D-2}\right)\left[\frac{m^{2}}{g^{2}(D-5)}\right]^{\frac{1}{D-5}},
$$

which corresponds (for $D \geq 6$ ) to a positive cosmological constant in the $(D-2)$-dimensional spacetime. (Note that (70) implies that the cosmological constant in the $(D+1)$-dimensional pure gravity theory is also positive.) This allows, in particular, a ground-state solution of the original $D$-dimensional theory of the form $M_{D-2} \times S^{2}$, where $M_{D-2}$ is an Einstein spacetime with positive cosmological constant, such as de Sitter space. Interpreted as a solution of the $(D+1)$-dimensional pure Einstein theory with positive cosmological constant, it becomes $M_{D-2} \times S^{3}$, since in this solution the 2 -form $\hat{F}_{(2)}$ in $D$ dimensions is a constant multiple of the volume-form of $S^{2}$, and thus the $S^{1}$ in the reduction from $(D+1)$ dimensions is the Hopf bundle over $S^{2}$.

\section{Conclusions and discussions}

In this paper, we have investigated the consistency of the Kaluza-Klein sphere reduction of the theory described by the Lagrangian (3), comprising gravity coupled to a $p$-form field

\footnotetext{
${ }^{12}$ The cosmological constant in $(D+1)$ dimensions breaks the scale-covariance that a theory of gravity and antisymmetric tensors has, and so one only gets $S L(n, \mathbb{R})$, and not $G L(n, \mathbb{R})$ from a $T^{n}$ reduction in this case (see [21]).
} 
strength and a dilaton in $D$ dimensions. Specifically, we have focussed our attention on those cases where the reduction Ansatz at least includes all the Yang-Mills fields of the $S O(n+1)$ gauge group.

We have shown that by including the dilaton in the higher-dimensional theory, the possibilities for consistent sphere reductions are extended somewhat, in comparison to the case where the higher-dimensional starting point comprises only gravity and a $p$-form field strength. Specifically, if no dilaton is included the only possibilities for consistent sphere reductions of the kind we are considering are those associated with the $S^{4}$ and $S^{7}$ reductions of $D=11$ supergravity, and the $S^{5}$ reduction of type IIB supergravity. With the dilaton included, we find that consistent $S^{2}$ reductions are possible for the case of a 2 -form in the higher dimension $D$, and that consistent $S^{3}$ and $S^{D-3}$ reductions are possible for the case of a 3-form in the higher-dimension. These reductions are possible starting from an arbitrary dimension $D$, provided that the strength of the dilaton coupling to the 2 -form or 3 -form field strength is chosen appropriately.

The previously-known consistent sphere reductions from $D=11$ with a 4 -form, and $D=10$ with a self-dual 5 -form, were associated with supersymmetric higher-dimensional theories. In the examples that we have obtained in this paper, supersymmetry is clearly not in general playing a rôle, since the higher-dimensional starting point can be a theory of gravity, a dilaton and a 2-form or 3-form in any arbitrary dimension. It is probably more appropriate, therefore, to characterise the theories that admit consistent sphere reductions by the fact that they have the unusual property of giving rise to lower-dimensional theories with certain enhanced global symmetry groups upon toroidal reduction on $T^{n}$. In particular, a necessary condition for a consistent $n$-sphere reduction that retains all the Yang-Mills fields of $S O(n+1)$ is that the global symmetry $G L(n, \mathbb{R})$ of a generic theory reduced on $T^{n}$ must be enhanced to a group whose compact subgroup contains $S O(n+1)$. These symmetry enhancements occur only in exceptional cases, when scalars coming from the toroidal reduction of metric "conspire" with scalars coming from the reduction of the $p$ form field strength to give an enhanced global symmetry group. It so happens that this same feature of symmetry enhancement is a central feature also in theories such as $D=11$ and type IIB supergravity, and their toroidal reductions.

It should be emphasised that the group-theoretic argument that we have been using in order to determine when a consistent sphere reduction may be possible does not, of itself, provide a guarantee of consistency.[3] Rather, it can be viewed as providing a proof of

\footnotetext{
${ }^{13}$ Unlike the traditional group theory argument that proves conclusively the consistency of a truncation
} 
inconsistency in cases where the necessary enhancement of the global symmetry group in the associated toroidal reduction does not occur. It is rather striking, however, that in all cases where a suitable sufficiently large global symmetry enhancement does occur, we find that a consistent sphere reduction is possible.

An interesting illustration of this point is provided by the reductions that we considered in section 7, where an additional "cosmological term" was included in the higher-dimensional theory. The argument based on global symmetry enhancement showed that the $S^{D-3}$ reduction would no longer be consistent, but that the $S^{3}$ and $S^{2}$ reductions still had the possibility of being consistent. And indeed this is just what we found, when we substituted the Ansätze into the equations of motion of the higher-dimensional theories with the cosmological terms included.

Although we have argued that supersymmetry is in some sense not of itself the directly crucial ingredient in the question of consistency, it is, nevertheless, worthwhile to consider further the question of supersymmetry and consistent sphere reductions. As well as the examples of the $S^{4}$ and $S^{7}$ reductions from $D=11$, and the $S^{5}$ reduction from $D=10$, we can now also consider those examples amongst the reductions constructed in this paper that can be associated with supersymmetric theories. Thus, for instance, we can consider the $S^{2}$ reduction of type IIA supergravity, using the R-R 2-form, and the $S^{3}$ and $S^{7}$ reductions of type I or type II supergravity, using the NS-NS (or R-R in the case of type IIB) 3-form.

Constructing the Kaluza-Klein sphere reduction Ansatz for the fermions in a supergravity theory is a notoriously difficult problem, and even when it is attempted the efforts are rarely extended to include the quartic fermion terms. However, we may construct a general argument to show that once a consistent reduction has been constructed in the bosonic sector, the supersymmetry of the higher-dimensional theory will then guarantee that a consistent reduction including the fermions as well must be possible. The argument is as follows. We know that a sphere reduction in which all fields (massive as well as massless) are retained will necessarily be consistent, and it will give rise to a supersymmetric lower-dimensional theory. Furthermore, we know that all the non-linear couplings between the various lower-dimensional fields will be organised, by virtue of the lower-dimensional supersymmetry, into supersymmetrically-covariant couplings of complete supermultiplets. Now, if we demonstrate in the bosonic sector that there is a consistent truncation to the massless sector (i.e. to the bosonic sector of the massless lower-dimensional supermultiplet), then this means that there are no interaction terms in which powers of the massless bosonic in which all singlets under a symmetry group are retained, and all non-singlets are truncated. 
fields (i.e. conserved currents built from the massless fields (see [32])) couple to linear powers of the massive bosons that are being set to zero. But this in turn implies that in the full theory there can be no interaction terms in which supercurrents built from the massless multiplet couple to linear powers of the massive fields. Thus if one shows that it is consistent to make a sphere reduction in which all the bosons of the massless supermultiplet are retained, then this implies that it must be consistent to make a sphere reduction of the supersymmetric theory in which the entire massless supermultiplet is retained.

One can use this argument to show that the $S^{3}$ and $S^{7}$ reductions of $N=1$ tendimensional supergravity, which are special cases of our more general results in this paper, will be consistent, as a consequence of our results for the bosonic sectors.

\section{Acknowledgement}

C.N.P. is grateful to the University of Pennsylvania for hospitality during the course of this work. We are grateful to Arta Sadrzadeh and Tuan Tran for extensive discussions.

\section{References}

[1] M.J. Duff and C.N. Pope, Kaluza-Klein supergravity and the seven sphere, in: Supersymmetry and supergravity 82, eds. S. Ferrara, J.G. Taylor and P. van Nieuwenhuizen (World Scientific, Singapore, 1983).

[2] P.G.O. Freund and M.A. Rubin, Dynamics of dimensional reduction, Phys. Lett. B97 (1980) 233.

[3] B. de Wit and H. Nicolai, N=8 supergravity, Nucl. Phys. B208 (1982) 323.

[4] B. de Wit and H. Nicolai, The consistency of the $S^{7}$ truncation in $D=11$ supergravity, Nucl. Phys. B281 (1987) 211.

[5] H. Nastase, D. Vaman and P. van Nieuwenhuizen, Consistent nonlinear KK reduction of 11d supergravity on $A d S_{7} \times S_{4}$ and self-duality in odd dimensions, hep-th/9905075.

[6] H. Nastase, D. Vaman and P. van Nieuwenhuizen, Consistency of the $A d S_{7} \times S_{4}$ reduction and the origin of self-duality in odd dimensions, hep-th/9911238. 
[7] M. Cvetič, M.J. Duff, P. Hoxha, J.T. Liu, H. Lü, J.X. Lu, R. Martinez-Acosta, C.N. Pope, H. Sati and T.A. Tran, Embedding AdS black holes in ten and eleven dimensions, Nucl. Phys. B558 (1999) 96, hep-th/9903214.

[8] P. Kraus, F. Larsen and S.P. Trivedi, The Coulomb branch of gauge theory from rotating branes, JHEP 9903 (1991) 003, hep-th/9811120.

[9] J.G. Russo and K. Sfetsos, Rotating D3-branes and QCD in three dimensions, Adv. Theor. Math. Phys. 3 (1991) 131, hep-th/9901056.

[10] M. Cvetič and S.S. Gubser, Phases of R-charged black holes, spinning branes and strongly coupled gauge theories, JHEP 04 (1999) 024, hep-th/9902195.

[11] M. Cvetič and D. Youm, Rotating intersecting M-branes, Nucl. Phys. B499 (1997) 253 , hep-th/9612229.

[12] H. Lü and C.N. Pope, Exact embedding of $N=1, D=7$ gauged supergravity in $D=11$, Phys. Lett. B467 (1999) 67, hep-th/9906168.

[13] H. Lü, C.N. Pope and T.A. Tran, Five-dimensional $N=4 S U(2) \times U(1)$ gauged supergravity from type IIB, Phys. Lett. B475 (2000) 261, hep-th/9909203.

[14] M. Cvetič, H, Lü and C.N. Pope, Four-dimensional $N=4$, $S O(4)$ gauged supergravity from $D=11$, hep-th/9910252, to appear in Nucl. Phys. B.

[15] M. Cvetič, H. Lü and C.N. Pope, Gauged six-dimensional supergravity from massive type IIA, Phys. Rev. Lett. 83 (1999) 5226, hep-th/9906221.

[16] M. Cvetič, S.S. Gubser, H. Lü and C.N. Pope, Symmetric potentials of gauged supergravities in diverse dimensions and Coulomb branch of gauge theories, hep-th/9909121, to appear in Phys. Rev. D.

[17] M. Cvetič, H. Lü, C.N. Pope and A. Sadrzadeh, Consistency of Kaluza-Klein sphere reductions of symmetric potentials, hep-th/0002056.

[18] M. Cvetič, H. Lü, C.N. Pope, A. Sadrzadeh and T.A. Tran, Consistent SO(6) reduction of type IIB supergravity, hep-th/0003103.

[19] E. Cremmer, Supergravities in 5 dimensions, in "Superspace and Supergravity", Eds. S.W. Hawking and M. Rocek (Cambridge Univ. Press, 1981) 267. 
[20] B. Julia, Group disintegrations, in "Superspace and Supergravity", Eds. S.W. Hawking and M. Rocek (Cambridge Univ. Press, 1981) 331.

[21] E. Cremmer, B. Julia, H. Lü and C.N. Pope, Dualisation of dualities, Nucl. Phys. B523 (1998) 73, hep-th/9710119.

[22] E. Cremmer, B. Julia, H. Lü and C.N. Pope, Higher-dimensional origin of $D=3$ coset symmetries, hep-th/9909099.

[23] H. Nastase and D. Vaman, On the non-linear KK reductions on spheres of supergravity theories, hep-th/0002028.

[24] H. Lü, C.N. Pope, E. Sezgin and K.S. Stelle, Stainless super p-branes, Nucl. Phys. B456 (1995) 669, hep-th/9508042.

[25] M.J. Duff, R.R. Khuri and J.X. Lu, String solitons, Phys. Rep. 259 (1995) 213, hepth/9412184.

[26] S. Mizoguchi and N. Ohta, More on the similarity between $D=5$ simple supergravity and M-theory, Phys. Lett. B441 (1998) 123, hep-th/9807111.

[27] A. Sen, Strong-weak coupling duality in four-dimensional string theory, Int. J. Mod. Phys. A9 (1994) 3707, hep-th/9402002.

[28] A. Sen, Strong-weak coupling in three dimensional string theory, Nucl. Phys. B434 (1995) 179, hep-th/9408083.

[29] A.H. Chamseddine and W.A. Sabra, $D=7 S U(2)$ gauged supergravity from $D=10$ supergravity, Phys. Lett. B476 (2000) 415, hep-th/9911180.

[30] M. Cvetič, J.T. Liu, H. Lü and C.N. Pope, Domain wall supergravities from sphere reduction, Nucl. Phys. B560 (1999) 230, hep-th/9905096.

[31] A. Achúcarro and P.K. Townsend, A Chern-Simons action for three-dimensional anti-de Sitter supergravity theories, Phys. Lett. B180 (1986) 89.

[32] C.N. Pope and K.S. Stelle, Zilch currents, supersymmetry and Kaluza-Klein consistency, Phys. Lett. B198 (1987) 151.

[33] C.G. Callan, D. Friedan, E.J. Martinec and M.J. Perry, Strings in background fields, Nucl. Phys. B262 (1985) 593. 